\title{
A Reliability Study of the Transmission Expansion Plan of Ecuador
}

\author{
Hellen Alvarez*, Ricardo Capaje ${ }^{\dagger}$, Juan Plazarte ${ }^{\ddagger}$, and Javier Urquizo ${ }^{\S}$ \\ Escuela Superior Politécnica del Litoral, ESPOL, FIEC \\ Campus Gustavo Galindo Km 30.5 Vía Perimetral, Guayaquil, Ecuador \\ Email:*helanalv@espol.edu.ec, ${ }^{\dagger}$ rcajape@espol.edu.ec,,${ }^{\S}$ jurquizo@espol.edu.ec \\ TRANSELECTRIC, Departamento de Planificación de la Expansión \\ Avenida 6 de Diciembre y Orellana, Quito, Ecuador \\ Email: ${ }^{\dagger}$ juan.plazarte@celec.gob.ec
}

\begin{abstract}
The objective of this article is to evaluate the reliability of the Ecuadorian National Interconnected System (acronym in Spanish SNI). It focuses on the sufficiency of the network and considers the projected changes in accordance with the proposals for the expansion of the transmission of the Electrification Master Plan for 2018 and 2025, under conditions of maximum demand. Two alternative methods were proposed for this analysis: the Exhaustive State Enumeration, and a Monte Carlo probabilistic simulation. As a decision parameter for the choice of one of these methodologies, we used the results of a preliminary test on the IEEE fourteen bus standard model, and determined that the best method for the evaluation of the national system was the Monte Carlo simulation. Using this method, our results demonstrated the reliability status of the SNI. The key theoretical elements for the quantification of $r$ eliability c orrespond $t$ ot he reliability indexes, and we consider the energy index of reliability (EIR), the loss of load expected value (LOLE), the loss of load probability (LOLP), the expected power not supplied (EPNS), the expected energy not supplied (EENS), the frequency of interruption (FOI), and the duration of interruption (DOI). These data were obtained for the years 2018 and 2025, by analysing the transmission system and the composite level of transmission generation for a period of 20 years, with convergence criteria of $0.05 \%$. Finally, the computational tool proposed within the development of this project helped determine that the plan of action exposed in the Electrification $M$ aster $P$ lan $d$ oes $h$ elp $t o$ i mprove $t$ he reliability of the system between the years of study. However, based on the analysis of the very high numerical values seen in the reliability indexes, it should be noted that, although Ecuador is in the process of improving its electrical system, improvements will still be needed beyond 2025 . This indicates that it is advisable to continue to conduct studies on the problems of the currently developing Ecuadorian system.
\end{abstract}

Keywords - Reliability analysis, Energy reliability index, State Enumeration Method, Monte Carlo Simulation Method, Loss of load probability, Expected power not supplied

\section{INTRODUCTION}

The continuous growth of the Ecuadorian population is reflected in the increase of resources consumption, emphasizing the increase in energy needs. Between 1999 and 2010, the number of inhabitants in the country grew from approximately $12,121,000$ to $14,307,000$; likewise, the demand for electric power during this period increased from $7,731 \mathrm{GWh}$ to $14,182 \mathrm{GWh}$ [6], which implies a population increase of around $18 \%$, whilst the energy demand was very close to doubling itself. In 2016, the country's energy demand was 27,154 $\mathrm{GWh}$, of which 23,518 GWh were for the National Interconnected System (SNI) [1]. Considering 2016 as the base year and the trend

Digital Object Identifier (DOI):

http://dx.doi.org/10.18687/LACCEI2020.1.1.274

ISBN: 978-958-52071-4-1 ISSN: 2414-6390 for growth in demand, it is estimated that the quantified need for energy by 2025 will reach an average value of 37,912 GWh. It is therefore necessary to adapt the national electrical system to the new requirements, giving rise to projects such as those described in the Electrification Master Plan [1]. This plan proposes the expansion of parts of the SNI, such as generation, transmission, and distribution, due to the projections for growth in electricity demand in different population settlements. It is hoped that this expansion will enable Ecuador to improve the utilisation of its territorial resources [1]. Figure 1 provides a summary of selected projects included in the transmission expansion plan.

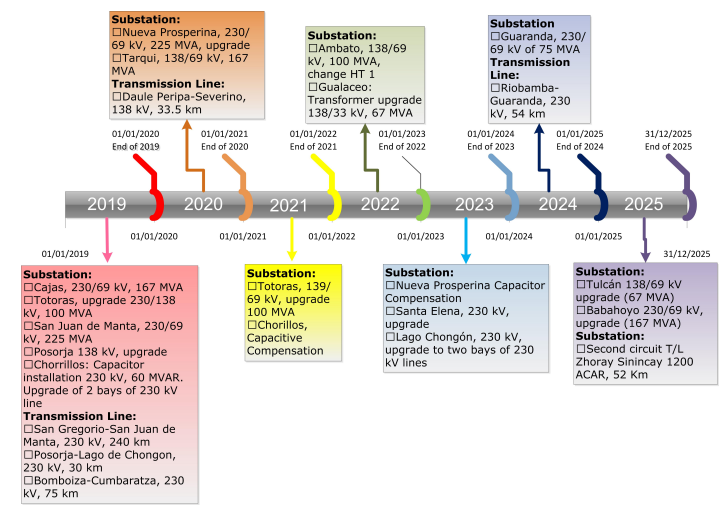

Fig. 1: Summary of the expansion plan of the transmission between 2019 and 2025 made from the data of the expansion plan of the Electricity Master Plan (PME) [1].

The expansion of the transmission will involve the manipulation of the lines and substations forming the National Interconnected System of Ecuador. Figure 2 details the planning of the expansion of the electrical generation between 2019 and 2025. It can be noted that the expansion of electrical generation does not occur for all the years of the study period, but only for the years 2022, 2023, and 2025. This is due to the extension of the period, which involves the implementation of electrical generation works, unlike the expansion of the transmission projects from 2019 to 2025.

One of the objectives of the expansion plan described above is to guarantee a continuous electric power service to the population, even in the face of contingencies. However, it is not always possible to maintain the energy service in all areas of the system because it is committed to faults of random origin, which in many cases are beyond the control of the operator. For this reason, there is a need to improve the reliability of the system through simulation studies. Due to the agreements between Transelectric S.A. (referred as Transelectric) and ESPOL, this reliability analysis of the National Interconnected System of Ecuador has focused on the sufficiency of 


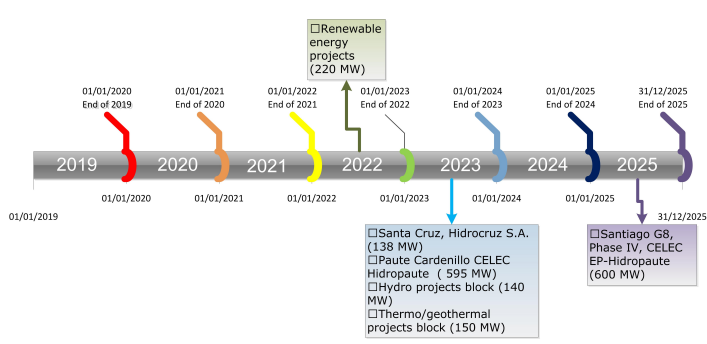

Fig. 2: Summary of the generation expansion plan between 2019 - 2025, according to information from the PME [1].

the system, according to the Electrification Master Plan 2016-2025 for 2018 and 2025. It considers the proposed transmission and generation systems, calculating reliability indexes (probability of loss of load, expected load loss, power not supplied, expected energy not supplied, energy reliability index, failure frequency and duration of failure), for the evaluation of the influence of the planned expansion on the improvement of the reliability indexes of the system. For this purpose. This paper focuses on a hitherto unexplored research questions, for which at present there is no definitive answer, which in essence relate primarily to the issues of reliability analysis in emerging countries where two reliability analysis methodologies are possible: the first is based on the modelling of states where the analytical probabilistic method of State Enumeration is found, and the second is supported by a simulation such as the Monte Carlo method. Initially, the development of both methodologies was proposed, and they were applied as preliminary tests in the IEEE fourteen bus system. However, we aimed to select the method that best suits the needs of the National System, using the PowerFactory platform through DIgSILENT Programming language development (DPL). Through this investigation, the Monte Carlo method was chosen for the study of the National Interconnected System. This is because the number of elements involved in the analysis causes the State Enumeration method to correspond to a process where more simulations can be executed in comparison to the Monte Carlo method, which, thanks to its convergence criteria, can find a solution more quickly. For our purposes, the present project is divided into six sections. The first introduces the project; the second indicates the methodology used for the development of the project; the third shows the results obtained through the application of the chosen logic; the fourth discusses the results obtained and the processes chosen; the fifth thanks the collaborators of the investigation; and, finally, the sixth indicates the references and works used as a guide for this investigation.

\section{METHODOLOGY}

For this project, we obtained a database with which to model the National Interconnected System (SNI) in PowerFactory DIgSILENT from the ESPOL-Transelectric, and information on the behaviour of the elements of the SNI. Some of this information for the second database was obtained from the same source, but two other sources which will be detailed later also supplied information. Next, the development of two methodologies for the reliability analysis in the DIgsSILENT program was proposed. Subsequently, the implementation of the scripts in the IEEE fourteen bus system was carried out to verify that the logic proposed for the methodologies coincided. This was done by comparing their indexes, in addition to supporting the choice of one of the methods for the SNI. Following this, the Monte Carlo method was chosen for the analysis of the SNI for 2018 and 2025, to obtain its reliability indexes by analysing both the transmission system and the transmission generation compound system under conditions of maximum demand in the dry season. The concepts and considerations used during the development of the research project will now be detailed.

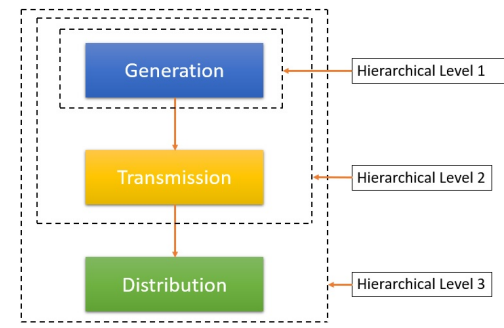

Fig. 3: Hierarchical levels for the analysis of power systems [2].

\section{A. System Modelling}

To simplify the perception of power systems, they are divided into functional zones: generation, transmission, and distribution. This facilitates the operation of the system and its planning [2]. Sometimes, it is necessary to analyze the interaction between the zones, causing the creation of "Hierarchical Levels" (Figure 3). As the hierarchical level increases, so does the number of zones covered. The main objective of this project is to evaluate the effect of transmission expansion, which is why transmission zone and hierarchical level 2 , which includes transmission and generation, are considered. The elements that form the power systems present a certain degree of availability based on the historical behaviour, and it was established that each element can only present two possible states: available and unavailable [3]. The transition between both states is in accordance with their average exit rate and average rate of restoration or repair (Figure 4).

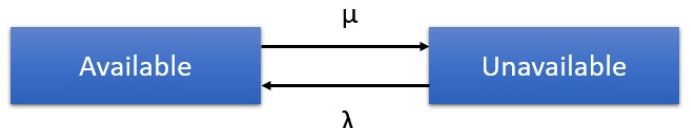

Fig. 4: States of availability of an element [3].

Where the failure rate $(\lambda)$ and the repair rate $(\mu)$ are obtained from Equations 1 and 2.

$$
\begin{aligned}
\lambda & =\frac{1}{\text { Available Hours }} \\
\mu & =\frac{1}{\text { Unavailable Hours }}
\end{aligned}
$$

Based on the Markov model, the transition time between each state is given according to the transition rates of each element [3].

$$
\begin{aligned}
& A=\frac{\mu}{\lambda+\mu} \\
& U=\frac{\lambda}{\lambda+\mu}
\end{aligned}
$$

There is the probability of being in the operating state (A) or failure state (U), according to Equations 3 and 4 . The methods of analysis to be used are defined below.

\section{B. State Enumeration Method}

The State Enumeration examines all possible contingency scenarios in a system, obtaining its probability of occurrence [4]. The number of states for an analysis of order n-2 (up to two elements in failure) is obtained from Equation 5, where $\mathrm{n}$ corresponds to the number of components.

$$
\text { Number of States }=\frac{n !}{2 !(n-2) !}+n+1
$$


Initially the database is collected with information about the unavailability and availability of each element, we then proceed to the calculation of the failure and repair rates; and the probability of operation and failure is obtained as mentioned above. Subsequently, the Contingency Matrix is prepared. From this we obtain the State Matrix that will allow us to calculate the probabilities by state and determine if there is energy loss for each scenario, finally the reliability index can be obtained.

The Contingency Matrix is obtained from matrices A and B. Matrix A contains the number of repeating elements per column, meaning that the cells of all the rows in column 1 will have the number 1 , likewise all rows from column 2 will have the number 2 , until obtaining a square matrix where its dimension will correspond to the number of elements $x$ number of elements.

Matrix B is a upper triangular type matrix, where by row the number of elements is completed by eliminating the minor ones and replacing the final value with 0 until completing a square matrix of dimension number of elements $\mathrm{x}$ number of elements. For $\mathrm{n}$ elements, row 1 of column 1 will have the value 1 and row $n$ of column 1 will have the value n. For column 2, row 1 will have the value of 2 , row $\mathrm{n}$ will have the value of 0 , while row $\mathrm{n}-1$ will contain the value $\mathrm{n}$; until you reach column $n$ where only row 1 will have the value of $n$, while the other rows will be filled with 0 .

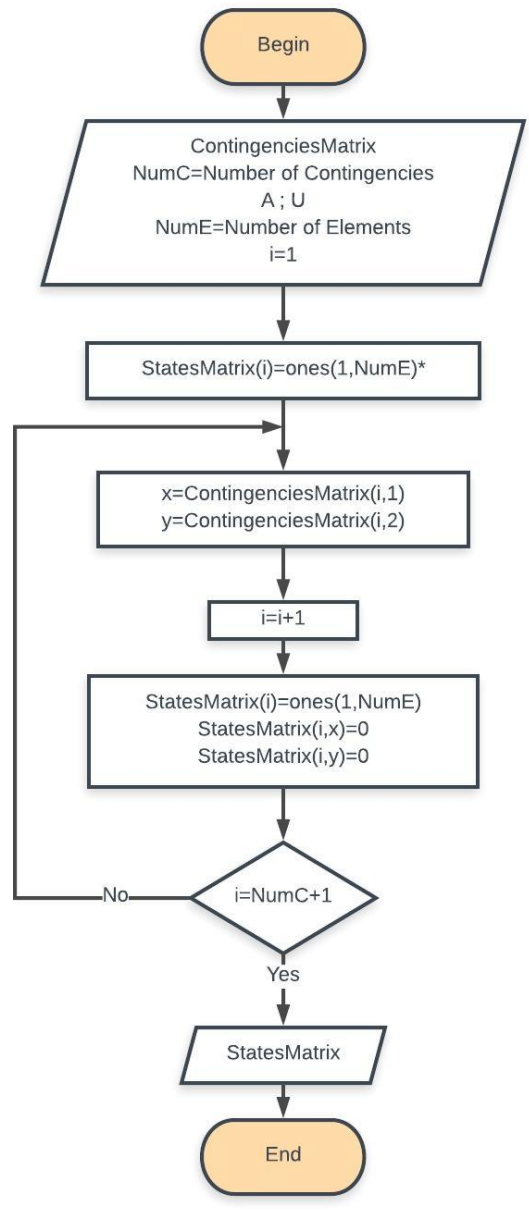

Fig. 5: Flow diagrams for the creation of the State Matrix in the State Enumeration Method.

Next, we created a Contingency Matrix, in which we compared each cell of Matrix A with that of the same position of Matrix $\mathrm{B}$; if both values coincide and are different from zero, then it is a contingency of the order $\mathrm{n}-1$; if the values do not coincide and are different from zero, it is a contingency of the order $n-2$. If any of the values of the cells is equal to zero, then it is not considered a contingency. Each pair of values is placed in one of the rows of the Contingency Matrix, so there is a matrix of two columns and a number of rows, coinciding with the number of contingencies.

This States Matrix and the steps in its creation can be seen in the flow diagram indicated in Figure 5. Initially, a matrix of ones is created, and then a sweep is made on the Contingency Matrix. For each iteration, the values of the pair of its columns were obtained and columns of the States Matrix that match to those values were looked for, and the 1 was replaced with a zero. The zero indicates that the element is unavailable and 1 refers to that it is available.

Finally, we executed a DC flow for each state, and when this action was complete the reliability indexes are calculated according to the following equations [4]

- Loss of Load Probability.

$$
L O L P=\sum P_{E N S}
$$

- Loss of Load Expected.

$$
L O L E=L O L P \times 8760[\text { hours } / \text { year }]
$$

- Expected Power Not Supplied.

$$
E P N S=\sum\left(\text { Power } N S \times P_{E N S}\right)[M W / \text { year }]
$$

- Expected Energy Not Supplied.

$$
E E N S=E P N S \times 8760[M W h / \text { year }]
$$

- Expected Energy Not Supplied in per unit.

$$
\text { EENSpu }=\frac{E E N S}{\text { Demanda Total } \times 8760}
$$

- Energy Index of Reliability.

$$
E I R=1-E E N S p u
$$

The indexes describe the effects of network failures.

\section{Montecarlo Method}

This method simulates the states of the system using iterative processes, which generate random numbers [5]. For the present work, a sequential, mixed-time logic was used [6]. Initially, a database was created and the failure and repair rates calculated; random numbers were generated that define the duration of the fault and operation states of each element, according to Equations 12 and 13.

Mean Time to Failure (MTTF)

$$
M T T F=-\frac{1}{\lambda} \ln U
$$

Mean Time to Repair (MTTR)

$$
M T T R=-\frac{1}{\mu} \ln U
$$

The operation sequence of each component is this obtained, since the elements of the system have different failure and repair rates, some will arrive before the set analysis time limit (Figure 6).

Each time the simulation reaches the set time, the system is evaluated in general, analysing the failure states per element, and identifying which coincide; hence, it gives the general failure in the system and determines the contingencies that caused a loss of energy (Figure 7).

Subsequently, an analysis of the results of the DC flow is obtained, giving information on the calculation of reliability indexes that will be used to validate the convergence criterion defined with the errors of the expected value indexes of non-supplied power and the probability of loss indexes which will be used to validate the convergence criteria. This is defined as the errors of the expected value indexes of unsupplied power, and the probability of loss of load. 


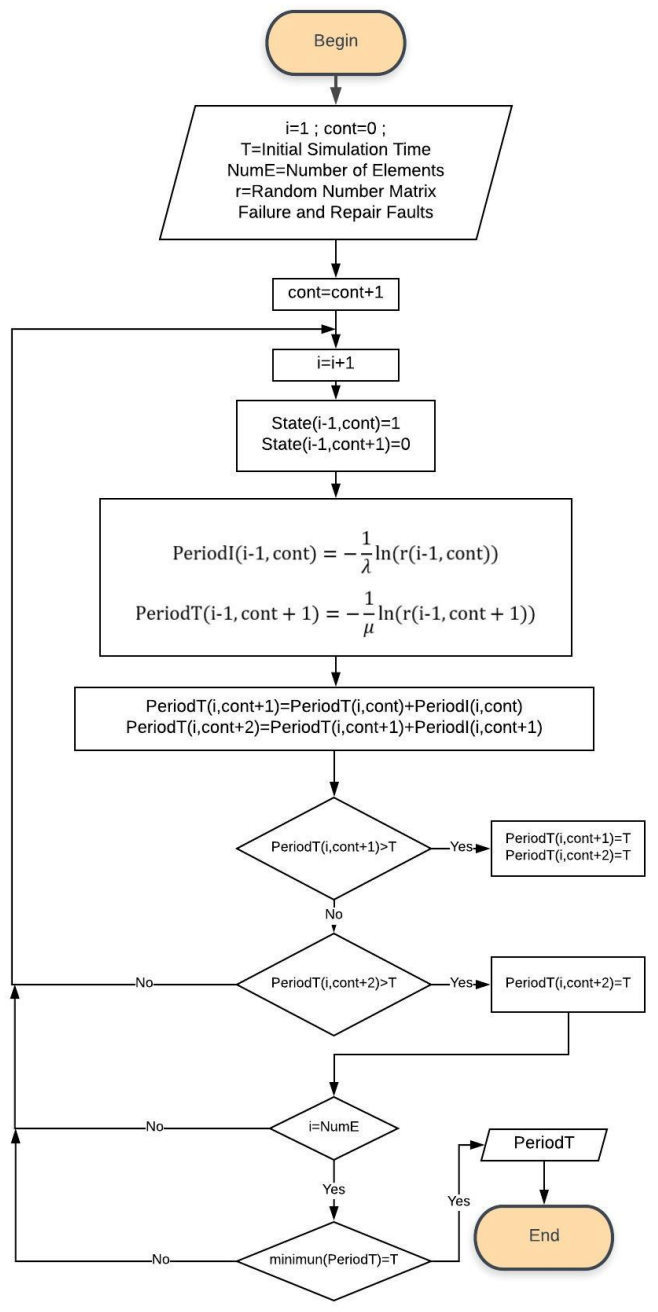

Fig. 6: Flow diagrams for obtaining the matrices of state and times of the elements of the system.

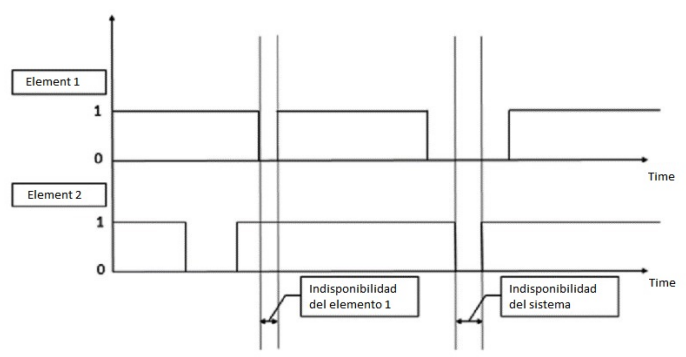

Fig. 7: Identification of the contingencies of the elements and failure of the power system in general.

- Loss of Load Probability.

$$
L O L P=\frac{\text { Time in which there was ENS }}{\text { Total time of simulation }}
$$

- Loss of Load Expected.

$$
L O L E=L O L P \times 8760[\text { hours } / \text { year }]
$$

- Expected Power Not Supplied.

$$
E P N S=\frac{P N S \text { during simulation }}{\text { Total time of simulation }}[M W / \text { year }]
$$

- Expected Energy Not Supplied.

$$
E E N S=E P N S \times 8760[M W h / \text { year }]
$$

- Expected Energy Not Supplied per unit.

$$
\text { EENSpu }=\frac{\text { EENS }}{\text { Total Demand } \times 8760}
$$

- Energy Index of Reliability.

$$
E I R=1-E E N S p u
$$

- Frequency of Interruption

$$
F O I=\frac{\sum \text { Interruptions }}{\text { Total time of simulation }}[\text { int } / \text { year }]
$$

- Duration of Interruption

$$
D O I=\frac{L O L E}{F O I}[\text { hours/intxyear }]
$$

These indexes describe the behaviour of faults and their effects on the network.

\section{Considerations for the analysis of systems}

For the IEEE system, the model included in DIgSILENT was chosen (Figure 8).

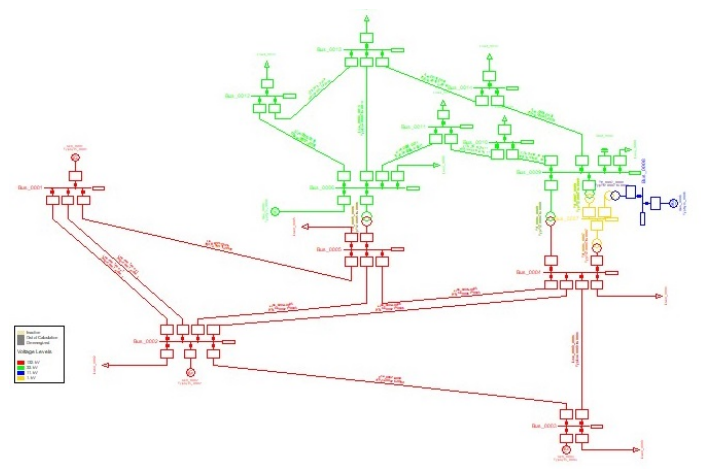

Fig. 8: IEEE fourteen bus system in DIgSILENT.

This analysis considered 21 lines and generators in total. The data on time of operation and failure were in the database made available by Transelectric. For the SNI, a filter was carried out to validate the following:

- Transmission lines with a voltage level greater than or equal to $69 \mathrm{kV}$, and a length greater than $10 \mathrm{~km}$;

- Transformers whose high voltage side have a nominal value greater than or equal to $138 \mathrm{kV}$;

- all generation units.

So, you would have the components that are indicated in Table 1.

To calculate the non-supplied power, loads greater than 5 MW were considered. With respect to the configuration of the DC flow, the 'Auto Slack' option from DIgSILENT was changed to "Method 2" (Figure 9).

This prevented the system's reference bar from being redefined by isolated systems; in other words, if a section is disconnected from the system, even it is formed by a generator and a load, the generator cannot be reprogrammed as slack, and therefore the isolated load will not be supplied. 
TABLE I: Summary of the number of SNI filtered elements for $2018-2025$

\begin{tabular}{|c|c|c|}
\hline \multicolumn{3}{|c|}{ National Interconnected System of Ecuador } \\
\hline Filtered elements & $\mathbf{2 0 1 8}$ & $\mathbf{2 0 2 5}$ \\
\hline $\begin{array}{c}\text { Lines }(500 \mathrm{kV}-69 \mathrm{kV}, \\
\text { length }>10 \mathrm{~km})\end{array}$ & 196 & 242 \\
\hline $\begin{array}{c}\text { Transformers with three } \\
\text { windings }(>138 \mathrm{kV})\end{array}$ & 94 & 119 \\
\hline Generators & 129 & 174 \\
\hline TOTAL & $\mathbf{4 1 9}$ & $\mathbf{5 3 5}$ \\
\hline
\end{tabular}

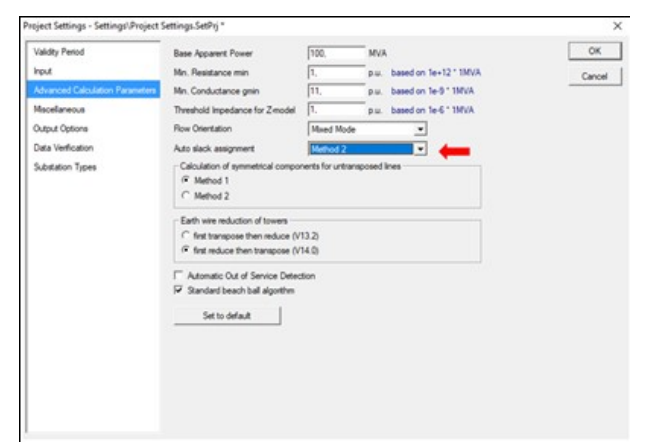

Fig. 9: Configuration of DC Flow in DIgSILENT.

\section{iII. Simulation Results}

In this section, we present the results of the simulations. Mainly, this information refers to two types of characteristic obtained as output data. The first type of output data corresponds to the effects that the failures had on the system, or, how the system reacted in a stable state. We obtained:

- Energy that could not be supplied for a year due to the failures experienced by the system (EENS);

- The value of the power associated with the non-supplied energy mentioned above (EPNS);

- The time in which there was a deficiency in the energy service towards the loads due to the failures (LOLE);

- The probability that demand exceeds electrical generation due to failures (LOLP);

- The energy index of reliability (EIR).

The second type of output data corresponds to the behavioural characteristics of the failures during the test period, as mentioned below:

- The frequency with which system failures (FOI) were experienced;

- The duration of the failures during the determined period (DOI).

As mentioned above, the State Enumeration method can only provide the first type of data since it does not seek to simulate the behaviour of the system; rather, it tries to list all possible scenarios, no matter how likely they may be. In contrast, the Monte Carlo simulation method provides more complete information, allowing us to access both types of data, since it seeks to simulate the behaviour of the elements of the system randomly based on their previous behaviour over a period of time.

To show the results obtained from the simulations, this section is divided into two subsections. Subsection 3.1 will show the results obtained from the IEEE fourteen bus system simulation, while Subsection 3.2 contains the results of the simulations of the SNI for the study of the transmission system and transmission generation system.

\section{A. Analysis of the IEEE fourteen bus system}

During the analysis of the IEEE system of fourteen bars the transmission-generation composite system was considered, it was
TABLE II: Results of the reliability indexes of the IEEE fourteen bus System in DIgSILENT

\begin{tabular}{|c|c|c|c|}
\hline \multicolumn{4}{|c|}{ IEEE System of fourteen Bus } \\
\hline $\begin{array}{c}\text { Reliability } \\
\text { Index }\end{array}$ & Units & $\begin{array}{c}\text { Enumeration } \\
\text { of States }\end{array}$ & Montecarlo \\
\hline LOLP & $\mathrm{pu}$ & 0,026763 & 0,026904 \\
\hline LOLE & $\mathrm{h} /$ year & 234,44 & 235,68 \\
\hline EPNS & MW/year & 6,928744 & 6.958831 \\
\hline EENS & MWh/year & 60695 & 60959,36 \\
\hline EENSpu & $\mathrm{pu}$ & 0,026752 & 0,02686807 \\
\hline EIR & $\mathrm{pu}$ & 0,973248 & 0,9731319 \\
\hline FOI & int/year & - & 1,318889 \\
\hline DOI & $\begin{array}{c}\text { h/int } \mathrm{x} \\
\text { year }\end{array}$ & - & 178,0691 \\
\hline
\end{tabular}

decided to implement the two methodologies previously exposed: State Enumeration and Montecarlo simulation. During the analysis of the IEEE fourteen bus system, the transmission generation composite system was considered, and it was decided to implement the two methodologies previously explained: the State Enumeration, and Monte Carlo simulation.

In Table 2, the results of the reliability indexes for both simulations are specified. For the analysis of the Enumeration of States, contingencies of the order $\mathrm{n}-2$ were considered, while for the Monte Carlo simulation a convergence criterion of $0.05 \%$ was considered, developed during 30 periods of 50 years each. Because the values obtained in Monte Carlo will only be compared with the results of State Enumeration, it is not necessary to show the convergence graphs for each of the reliability indexes during the IEEE system simulation.

It can be seen that the results obtained with both simulations were very close; for the value of the LOLP, there was a difference of $0.000141 \mathrm{pu}$; for the LOLE, the difference was $1.24 \mathrm{~h} /$ year; for the EPNS, there was a difference of $0.030087 \mathrm{MW} / \mathrm{year}$, which is reflected in a difference of $264.36 \mathrm{MWh} /$ year of EENS; likewise, there was a difference of 0.000109 pu for the EIR; and, as can be seen only in the Monte Carlo simulation, there was a value of 1.318889 int/year for the FOI and $178.07 \mathrm{~h} /$ intxyear for the DOI.

\section{B. Analysis of the SNI of Ecuador}

For the analysis of the SNI of Ecuador, evaluations were made considering two types of systems, the first corresponding only to the transmission system, and the second for the transmission and generation system. Both considerations were implemented for the two study periods of the years 2018 and 2025, according to the expansion projects described in the Electrification Master Plan [1] for the generation, transmission, and projection of demand as mentioned in the Introduction.

Under these conditions, during the analysis of the transmission only system for the year 2018, 290 elements were considered, while for 2025 there were 361 elements, including transmission lines and transformers with three windings. Likewise, for the analysis of the composite system for 2018, 419 elements were observed, while for 2025,535 were considered, including transmission lines, generators, and transformers with three windings. These numbers correspond to the number of filtered elements according to the validations described in the previous section.

Following Equation 5 for the calculation of the number of elements, and considering that the smallest number of elements involved in the analysis is for the transmission system of 2018, with 290 elements, 42,196 states need to be counted. This would involve a great deal of simulation time, and so it was decided to implement only the Monte Carlo simulation method for the analysis of the SNI. 


\section{Transmission System}

Next, we created the convergence graphs of the reliability indexes obtained from the analysis of the transmission system for 2018 and 2025, as shown by the Monte Carlo method. This was under an error convergence criterion of $0.05 \%$ for 30 periods of 50 years each, a total of 1,500 years. The simulation took approximately eight hours for each year of study. The red line represents the values obtained during the simulations for 2018, while the brown represents the results for 2025 .

\section{Energy Index of Reliability-SNI 2018 and 2025}

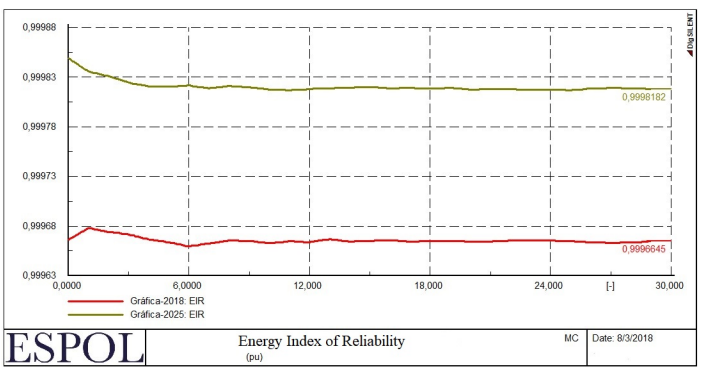

Fig. 10: Convergence of the EIR for the Ecuadorian SNI in 2018 and 2025.

Figure 10 shows that the reliability index for 2018 was 0.9996645 $\mathrm{pu}$, as shown by the red line, while for 2025 it was 0.9998182 pu (brown line). That is to say that, during 2018 the index of energy reliability was lower by 0.012932 in comparison to the value obtained for 2025 .

\section{Expected Power Not Supplied-SNI 2018 and 2025}

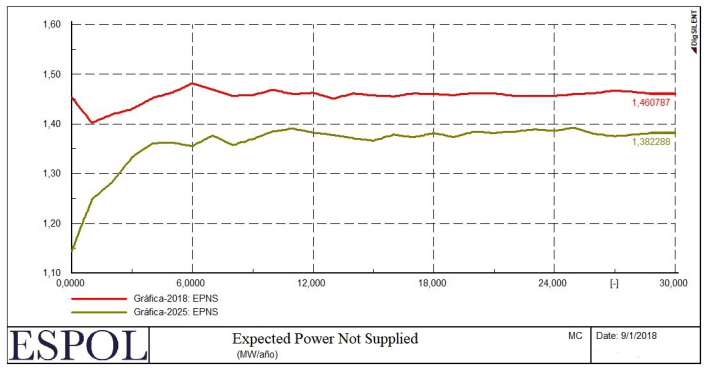

Fig. 11: Convergence of the expected value of the power not supplied for the Ecuadorian SNI in 2018 and 2025.

Figure 11 shows the convergence of the expected value of power not supplied. The red line indicates that for $20181.4608 \mathrm{MW} / \mathrm{year}$ was obtained, while the brown line shows that for 2025 it was 1.3823 MW/year. From 2018 until 2025, the value of the power not supplied decreased by $0.0785 \mathrm{MW} /$ year.

\section{Expected Energy Not Supplied-SNI 2018 and 2025}

Figure 12 shows the convergence behaviour of the expected value of non-supplied energy associated with the power not supplied. For 2018 , the convergence of the red line was $12796.50 \mathrm{MWh} / \mathrm{year}$, while for 2025 it was $12108.84 \mathrm{MWh}$ /year, shown in brown. In other words, from 2018 to 2025, the energy not supplied decreased by 687.66 MWh/year.

\section{Loss of Load Expected-SNI 2018 and 2025}

As it can be seen in the convergence graphs of Figure 13, the expected load loss value according to the red graph for the year

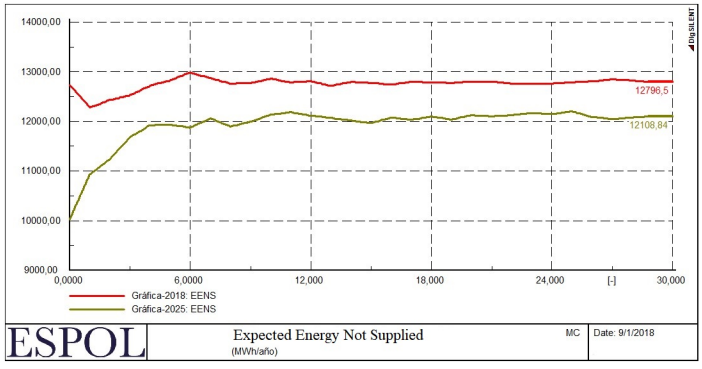

Fig. 12: Convergence of the expected value of the non-supplied energy for the Ecuadorian SNI in 2018 and 2025.

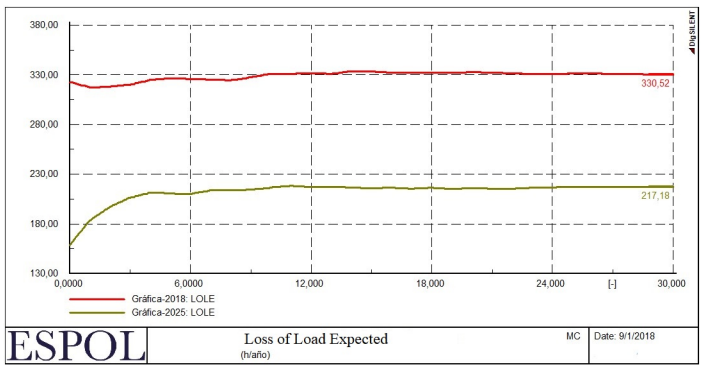

Fig. 13: Convergence of the expected value of load loss for the Ecuadorian SNI in 2018 and 2025.

2018 was $330.52 \mathrm{~h} /$ year, while according to the brown graph for the year 2025 it was of $217.18 \mathrm{~h} /$ year. That is to say that from 2018 to 2025 it decreased by $113.34 \mathrm{~h} /$ year.

\section{Loss of Load Probability-SNI 2018 and 2025}

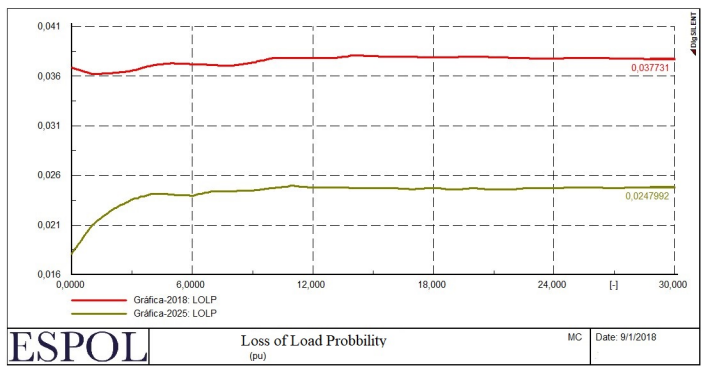

Fig. 14: Convergence of the probability of loss of charge for the Ecuadorian SNI in 2018 and 2025.

Figure 14 shows the convergence graph of the probability of loss of load. For 2018, a value of 0.037731 pu was obtained, shown in the red line, while the last value of the brown line for 2025 was $0.0247992 \mathrm{pu}$. In other words, the condition of this reliability index improves from 2018 to 2025 at $0.0129318 \mathrm{pu}$.

\section{Frequency of Interruption -SNI 2018 and 2025}

Figure 15 indicates that for 2018 there was an interruption frequency of six per year (red line), while for 2025 (brown) there were seven. This means that the frequency of interruption increases for 2025 by one per year.

\section{Duration of interruption-SNI 2018 y 2025}

As can be seen in Figure 16, the red graph line corresponding to 2018 converges to a value for interruption duration of 53.73 $\mathrm{h} /$ intxyear, while the brown line describes the behaviour for 2025 and 


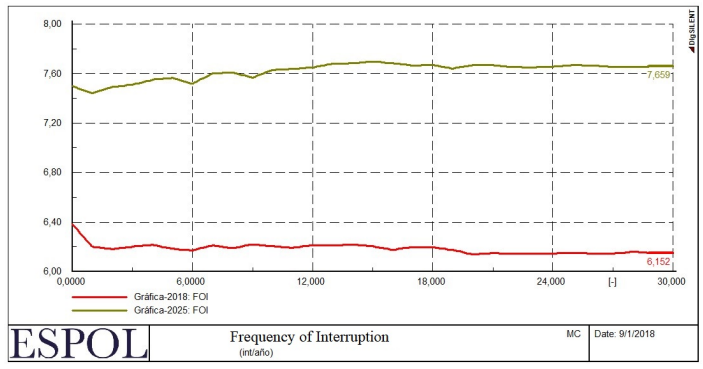

Fig. 15: Convergence of the frequency of interruption for the Ecuadorian SNI in 2018 and 2025.

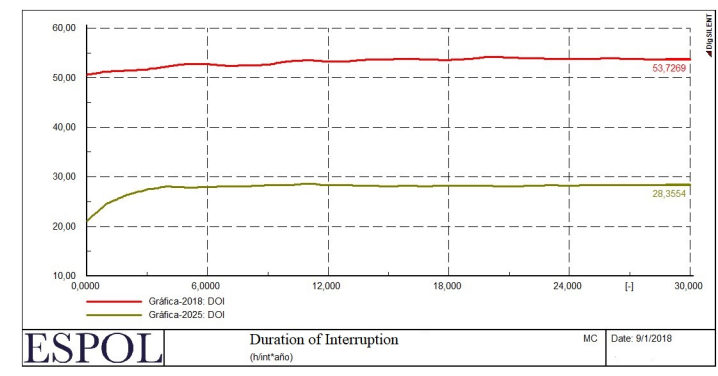

Fig. 16: Convergence of the interruption duration for the Ecuadorian SNI in 2018 and 2025.

shows a value of $28.36 \mathrm{~h} /$ intxyear. In other words, there is a decrease in the interruption duration for the year 2025 of $25.37 \mathrm{~h} /$ intxyear.

\section{Compound System: Generation-Transmission}

Below, the convergence graphs of the reliability indexes obtained from the analysis of the transmission and generation composite system for 2018 and 2025 are shown by means of the Monte Carlo simulation. As in the previous analysis, this is under a criterion of error convergence of $0.05 \%$ for 30 periods of 50 years each, which is a total of 1,500 years.

\section{Energy Index of Reliability-SNI 2018 and 2025}

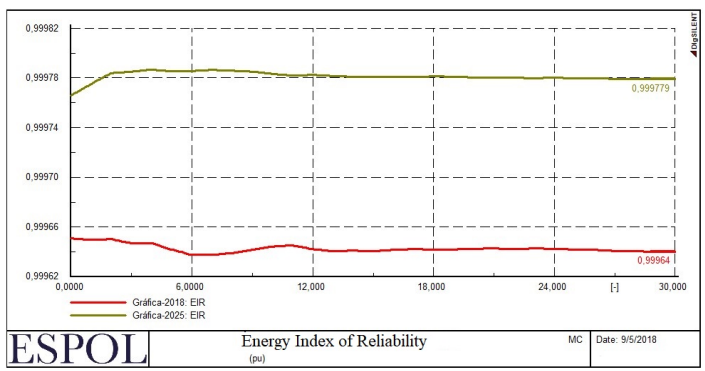

Fig. 17: . Convergence of the energy index of reliability for the Ecuadorian SNI in 2018 and 2025.

Figure 17 shows the convergence graph for the reliability index. The red graph line indicates that in 2018 the resulting value was $0.9999641 \mathrm{pu}$, while the brown shows that for 2025 the resulting value was $0.9999779 \mathrm{pu}$. During 2018 the reliability index of energy was therefore lower by 0.000138 pu compared to the value obtained for 2025 .

\section{Expected Power Not Supplied-SNI 2018 and 2025}

Figure 18 shows the convergence of the expected value of power not supplied, the red graph line indicates that for 2018 the value

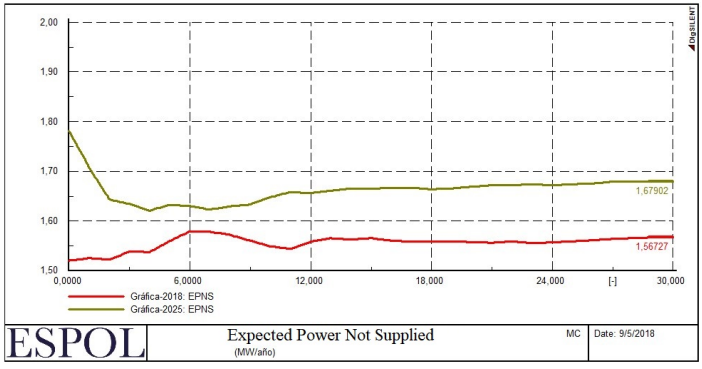

Fig. 18: Convergence of the expected value of the power not supplied for the Ecuadorian SNI in 2018 and 2025.

obtained was $1.56721 \mathrm{MW} /$ year, while the brown graph line shows that for 2025 it was $1.67902 \mathrm{MW} /$ year. From 2018 until 2025, the power not supplied thus increased by $0.11181 \mathrm{MW} / \mathrm{year}$.

\section{Expected Energy Not Supplied-SNI 2018 and 2025}

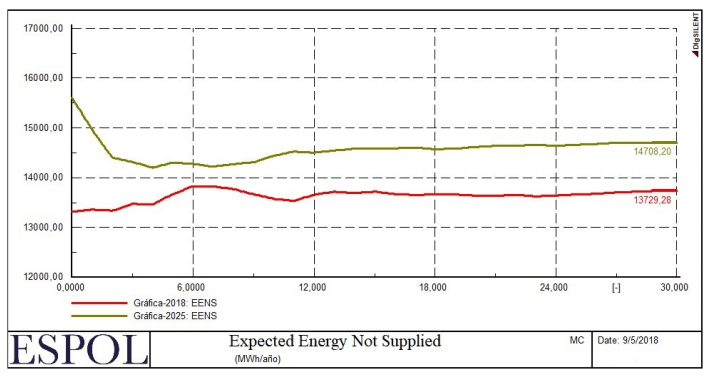

Fig. 19: Convergence of the expected value of the non-supplied energy for the Ecuadorian National

Interconnected System in 2018 and 2025.

Figure 19 shows the convergence behaviour of the expected value of non-supplied energy associated with the non-supplied power value reviewed in the previous point. The red graph line indicates that for 2018 there was an energy value not supplied of $13729.28 \mathrm{MWh} / \mathrm{year}$, while for 2025 (brown) there was a value of $14708.20 \mathrm{MWh} /$ year. In other words, from 2018 until 2025, the energy not supplied increased by $978.92 \mathrm{MWh} /$ year.

\section{Loss of Load Expected-SNI 2018 and 2025}

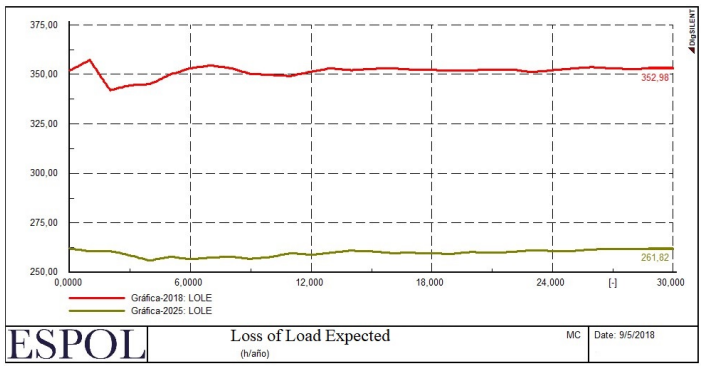

Fig. 20: Convergence of the expected value of load loss for the Ecuadorian SNI in 2018 and 2025.

As can be seen in the convergence graphs in Figure 20, the expected value of pressure loss for 2018 was $352.98 \mathrm{~h} /$ year, shown by the red line, while for 2025 it was $261.82 \mathrm{~h} /$ year (brown). Thus, from 2018 to 2025 it decreased by $91.16 \mathrm{~h}$ /year.

Loss of Load Probability-SNI 2018 and 2025 


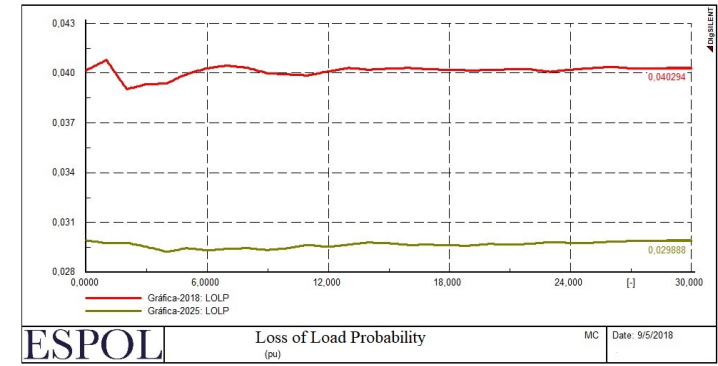

Fig. 21: Convergence of the probability of loss of charge for the Ecuadorian SNI in 2018 and 2025.

Figure 21 shows the convergence graph of the probability of loss of load for 2018 (red) and for 2025 (brown). For 2018, a value of 0.040294 pu was obtained, while for $2025,0.029888$ pu was obtained. The condition of this reliability index thus improves from 2018 to 2025 in $0.010406 \mathrm{pu}$.

\section{Frequency of Interruption -SNI 2018 and 2025}

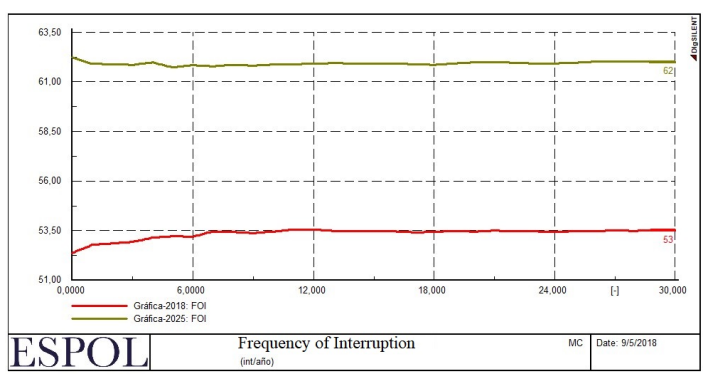

Fig. 22: Convergence of the interruption frequency for the Ecuadorian SNI in the year 2018 and 2025.

In terms of the interruption frequency, seen in Figure 22, for 2018 this had a value of 53 int/year (red), while for 2025 (brown) it had a value of $62 \mathrm{int} / \mathrm{year}$. This means that by 2025 the interruption frequency increased by $9 \mathrm{int} / \mathrm{year}$ in comparison to 2018

\section{Duration of interruption-SNI 2018 y 2025}

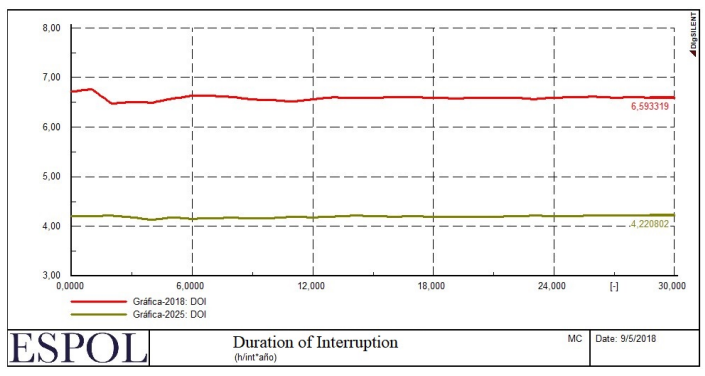

Fig. 23: Convergence of the interruption duration for the Ecuadorian SNI in 2018 and 2025.

As seen in Figure 23, the red line for 2018 shows a value for interruption duration of $6.5933 \mathrm{~h} /$ intxyear, while the brown line for 2025 shows a value of $4.2208 \mathrm{~h} / \mathrm{intxyear}$. There is therefore a decrease in the interruption duration for the year 2025 of $2.3725 \mathrm{~h} /$ intxyear.

\section{Summary of Results}

The results obtained from all the simulations described above in graphs of their convergence behaviour are summarized below. Tables
3 and 4 show the results for 2018 and 2025 during the analysis of the transmission system and the composite system, where the differences described above can be appreciated more easily.

TABLE III: Summary of results for 2018 and 2025 of the analysis of the transmission system

\begin{tabular}{|c|c|c|c|}
\hline $\begin{array}{c}\text { Reliability } \\
\text { Index }\end{array}$ & Units & $\mathbf{2 0 1 8}$ & $\mathbf{2 0 2 5}$ \\
\hline LOLP & $\mathrm{pu}$ & 0,037731 & 0,024799 \\
\hline LOLE & h/year & 330,52 & 217,18 \\
\hline EPNS & MW/year & 1,460787 & 1,382288 \\
\hline EENS & MWh/year & 12796,50 & 12108,84 \\
\hline EIR & pu & 0,9996645 & 0,9998182 \\
\hline FOI & int/year & 6 & 7 \\
\hline DOI & $\begin{array}{c}\text { h/int x } \\
\text { year }\end{array}$ & 53,7269 & 28,3554 \\
\hline
\end{tabular}

TABLE IV: Summary of results for 2018 and 2025 of the analysis of the composite system

\begin{tabular}{|c|c|c|c|}
\hline $\begin{array}{c}\text { Reliability } \\
\text { Index }\end{array}$ & Units & $\mathbf{2 0 1 8}$ & $\mathbf{2 0 2 5}$ \\
\hline LOLP & pu & 0,04029406 & 0,029888 \\
\hline LOLE & h/year & 352,98 & 261,82 \\
\hline EPNS & MW/year & 1,56727 & 1,699020 \\
\hline EENS & MWh/year & 13729,28 & 14708,20 \\
\hline EIR & pu & 0,999640 & 0,9997792 \\
\hline FOI & int/year & 53 & 62 \\
\hline DOI & $\begin{array}{c}\text { h/int } x \\
\text { year }\end{array}$ & 6,5933 & 4,2208 \\
\hline
\end{tabular}

Tables 3 and 4 show the results for 2018 and 2025 during the analysis of the transmission system and the composite system, where the differences described above can be appreciated more easily.

\section{DISCUSSION AND CONCLUDING OBSERVATIONS}

In this section, we discuss the results obtained from the reliability assessment of the SNI of Ecuador for 2018 and 2025, according to the data and the revised concepts that served to find potential solutions.

As mentioned initially, there were three main sources of data, corresponding to the historical behaviour of the filtered elements. The first source was the database of Transelectric, which gave failure times, repair times, and the forced interruption rate of transmission lines, bars and generators; however, given that the database did not include all the elements that form the SNI, and that in the present study the contingencies were not considered in bars, it was decided to use additional references: are Sánchez \& Tates [7] and Gorki [8]. These provided information on the values of frequency of faults, average time of failure, frequency of interruption, average time of interruption, and availability of some of the elements. Since the data come from different sources, it can be said that, in general, there is a random type error in the database. This is to say that there are approximate values to the real value; however, since the processes during the measurements cannot be controlled, it should also be noted that, even if all the data had been accessed from the same reference, the type of error would not change due to the random nature of the faults that control the operation, and the failure times necessary for the calculation of rates and subsequent declaration of states.

Through the literature review of the methods by which to study the reliability of power systems, we proposed Exhaustive State Enumeration and Monte Carlo simulation. Through the test of the standard IEEE fourteen bus system, it was possible to verify the Monte Carlo logic, since the approach to this is not as simple as that of the Enumeration of States. Because of the similarity of the 
results, it was possible to verify that the sequential process of Monte Carlo was adequate for the realization of the project. The main complications occurred in the handling of the data, which were treated so that the behaviour of the system could be modelled through a simulation time interval. This created the need to register the data in matrices and then use them in the identification of duration and simulation of faults, to obtain the energy not supplied in the system, and finally calculate the reliability indices for the analysis.

Our choice of the Monte Carlo simulation method of the mixedtime sequential type was adequate. The difference between the probabilities of operation and failure between the lines and the generators shows that the lines have lower repair rates. Therefore, they require fewer simulations to complete the simulation study of intervals in comparison with the generators, which causes a non-uniform advance. This creates the need for an independent consideration of each element's events, although at the same time a sequence of operating and out of service states for each element has to be created.

The SNI of Ecuador contains many elements that must be considered when carrying out a reliability analysis of the system. This complexity means that the most viable evaluation method is a Monte Carlo simulation, since it avoids the formulation of all the possible states that the number of base elements might have. Likewise, when the first Monte Carlo simulations were made, it could be observed that the longer the simulation time, the faster the reliability indexes converge. This is because when evaluating a larger interval, more states can be defined, and thus this models real behaviour more closely; however, the application of the failure and repair rates occurred in days; if the data were still considered in hours, as in the first tests, there would be many evaluation points and arriving at the convergence values of the reliability indexes would involve more simulation time and memory.

Analyzing the contents of Table 3, our research shows that the projections of the PME [6] help to improve the sufficiency of the network, since there is a decrease in the reliability indexes, with the exception of the energy reliability index, which also represents an improvement in the conditions of the system. However, analyzing Table 4, not all the indexes increase, and in this case the contingency in electrical generation significantly affects the sufficiency of the system. Nevertheless, it is necessary to emphasize that this is also due to the fact that the project considered the worst conditions, in which there is maximum demand or dry season. Moreover, the majority of electrical generation in Ecuador is from hydroelectric power plants.

Although improvements can be made according to our findings, the electrical system still has high values in its reliability indexes, which is why it still lends itself to studies that seek solutions to improve the difficulties of the SNI. It can also be seen that the analysis of the transmission system gives us a fairly close idea of the behaviour of the interconnected system, since the values obtained are close to those resulting from the analysis of the transmission generation system. This is also due to the fact that the implementation of the generation is weaker because there is greater investment in transmission expansion projects compared to the expansion of generation, although the demand continues to grow.

The limitations and recommendations for future research are mainly related to the form of programming in DPL, since it is not as versatile as other platforms dedicated to the programming of projects from different fields of study; it does not have facilities such as the creation of personalized functions, but routines and subroutines of different levels can be used which follow the order of a tree diagram, and whose communication is unilateral. That is to say, the data can only be sent from a higher level to a lower level, such as from a routine to a subroutine, but not vice versa; thus, if it is necessary to obtain the data of a subroutine for a recursive implementation with the generation of useful data for the continuation of the work of the routine, we recommend a dynamic database in Excel so that the variables of the routines call the information generated in the subroutines. Due to the processing capacity of DIgSILENT, this operation did not significantly affect the computational time used. Furthermore, a .txt file could also be used. In addition to this it should be noted that, although it is possible to send data from a routine to a subroutine as mentioned above, this information can only be of the entire variable type, double variable, character string, Object or Set, excluding in this case the matrices.

In summary, in this project we quantified the reliability of the Ecuadorian SNI, focusing on the generation and transmission systems, dedicated especially to the analysis of sufficiency of the demand in the country, by means of the creation of a program in the programming language of DIgSILENT (DPL). This serves as a basis for future reliability studies using the Monte Carlo simulation method, without limitations regarding the time of study or the degree of convergence error, and is applicable to other types of transmission generation systems, or alternatively it could only focus on the hierarchical level of transmission.

We show that, although it is expected that the reliability of the National System's sufficiency will improve according to the future implementations based on what is stated in the Electrification Master Plan for 2018 and 2025, it is necessary to carry out further studies in relation to sufficiency since the reliability indexes of the electric system of the country still present high values; adjustments could be made which, if necessary, can also be evaluated through the application of this project, serving as a backup for future decisions

\section{ACKNOWLEDGements}

We want to acknowledge the help received by the CELEC EP TRANSELECTRIC Business Unit, who allowed us to use their data sets. This was unique and invaluable in the development of this research

\section{REFERENCES}

[1] Ministerio de Electricidad y Energía Renovable, "Plan maestro de electricidad 2016-2025 [2016 - 2025 electricity master plan]," MEER, QuitoEcuador, Tech. Rep., 2017.

[2] R. Billinton and R. N. Allan, Reliability Evaluation of Power Systems, 2nd ed., Springer, Ed., Aug. 1996.

[3] C. Zapata, Confiabilidad de Sistemas Eléctricos de Potencia [Electrical Power Systems Reliability]. Universidad Tecnológica de Pereira, 2011. [Online]. Available: https://www.feis.unesp.br/Home/ departamentos/engenhariaeletrica/lapsee/curso_2011_zapata_2.pdf

[4] V. Fuentes and O. Duarte, Evaluación mediante enumeración de estados de la confiabilidad del Sistema Interconectado del Norte Grande de Chile (SING) [State enumeration approach in reliability assessment of the Chilean far north power system (SING)]. Universidad de Antofagasta de Chile, 2011. [Online]. Available: https://scielo.conicyt.cl/ scielo.php?script=sci_arttext\&pid=S0718-33052011000200013

[5] C. Zapata and E. Campos, "Valoración de confiabilidad de sistemas de generación con recursos limitados de energía utilizando simulación de montecarlo [reliability assessment of energy limited power generation systems using montecarlo simulation]," Scientia et Technica, vol. 29, 2005. [Online]. Available: https: //www.academia.edu/13962654/VALORACIN_DE_CONFIABILIDAD_ DE SISTEMAS DE GENERACIN CON RECURSOS LIMITADOS DE_ENERGA_UTILIZANDO_SIMULACIN_DE_MONTECARLO

[6] F. Imbarack, Elaboración de una herramienta computacional para la evaluación de la confiabilidad de sistemas de transmisión eléctricos [Elaboration of a computational tool for the evaluation of the electrical transmission systems reliability]. Pontificia Universidad Católica de Chile, 2006. [Online]. Available: http://hrudnick.sitios.ing. uc.cl/paperspdf/aarriagada.pdf

[7] G. Sánchez and D. Tates, "Análisis de confiabilidad del sistema nacional interconectado ecuatoriano utilizando el software neplan [reliability analysis of the ecuadorian national interconnected system using neplan software]," Escuela Politécnica Nacional, Quito - Ecuador, Tech. Rep., Nov. 2007. [Online]. Available: http://bibdigital.epn.edu.ec/handle/15000/ 4249

[8] G. Torres, "Adaptación de modelos para el cálculo de la confiabilidad del sistema nacional de transmisión para el año 2004, con el programa digsilent, aplicado a los agentes: Emelesa, termoesmeraldas $y$ eeq s.a. [adaptation of reliability computing models for the 2004 national transmission system with the digsilent program, applied to the agents: Emelesa, termoesmeraldas and eeq s.a.]," Escuela Politécnica Nacional, Quito - Ecuador, Tech. Rep., Nov. 2003. [Online]. Available: http://bibdigital.epn.edu.ec/handle/15000/5101 